\title{
Failure Mechanics of 2D Materials
}

\author{
Tobin Filleter \\ Department of Mechanical \& Industrial Engineering \\ University of Toronto, Canada
}

Over the last decade two-dimensional (2D) materials have emerged as a new class of advanced material due to their atomically thin geometries and extraordinary materials properties. In particular, 2D materials exhibit exceptional mechanical properties including some of the highest measured strength and stiffness found in nature. Since the discovery of freestanding graphene just over a decade ago, it and other 2D materials such as graphene oxide and tungsten disulphide (WS2) have been proposed for use in wide ranging mechanical applications including flexible sensors, solid/liquid lubricants, and lightweight composites. The use in such macroscopic engineering applications necessitates further studies of the failure mechanics of 2D materials beyond the initial studies of strength and stiffness. This talk will present recent advances in studying failure mechanics (i.e. fracture toughness \& fatigue) and tribology (i.e. friction, wear, lubrication) of 2D materials. 